\title{
Correction to: Cost-effectiveness of mass screening for dipstick hematuria in Japan
}

\author{
Reiko Okubo ${ }^{1,2,3} \cdot$ Shu-Ling Hoshi ${ }^{1} \cdot$ Tomokazu Kimura $^{4} \cdot$ Masahide Kondo $^{1}$ (i) $\cdot$ Koichi Asahi $^{5} \cdot$ Chiho Iseki $^{6}$. \\ Shouichi Fujimoto ${ }^{7} \cdot$ Ichiei Narita $^{8} \cdot$ Hiroyuki Nishiyama $^{4} \cdot$ Kunihiro Yamagata $^{2} \cdot$ Kunitoshi Iseki $^{6}$
}

Published online: 2 February 2022

(C) Japanese Society of Nephrology 2022

\section{Correction to: Clinical and Experimental Nephrology https://doi.org/10.1007/s10157-021-02170-0}

In the original publication, few errors were found in Figure 2c, Table 1 and Table 3 . The corrections are as follows:

1) In the Figure $2 c$

"3) Stage" should be "3)Stage I"

"4) "Stage I, Stage II, Stage III, Stage IV" is exactly "Stage II, Stage III, Stage IV"

Besides,"Stage I" should be separated from "Stage II, Stage III, Stage IV"

2) In the Table 1

1. We have added "**" to all 4 reference number 26 in Table 1.

(That is, the reference "[26]" has been changed to "[26*]".)

2. The line between SOa and SI in Male of Distribution of $\mathrm{BC}$ by clinical stage detected by screening by sex and age has been deleted.

3) In the Table 3

The original article can be found online at https://doi.org/10.1007/ s10157-021-02170-0.

Masahide Kondo

mkondo@md.tsukuba.ac.jp

1 Department of Health Care Policy and Health Economics, Faculty of Medicine, University of Tsukuba, 1-1-1

Tennoudai, Tsukuba, Ibaraki 305-8577, Japan

2 Department of Nephrology, Faculty of Medicine, University of Tsukuba, Tsukuba, Ibaraki, Japan

3 Department of Clinical Laboratory Medicine, University of Tsukuba Hospital, Tsukuba, Ibaraki, Japan

4 Department of Urology, Faculty of Medicine, University of Tsukuba, Tsukuba, Ibaraki, Japan
The "Variables" and "Base-case value" has been separated in first line.

That is, "Variables" should appear in the first column, and "Base-case value" in second column which should appear as below.

\begin{tabular}{ccc}
\hline Variables $\quad$ Base-case value & $\begin{array}{l}\text { Range tested in } \\
\text { sensitivity analy- } \\
\\
\text { sis (\%) }\end{array}$
\end{tabular}

The original article has been corrected.

Publisher's Note Springer Nature remains neutral with regard to jurisdictional claims in published maps and institutional affiliations.

5 Division of Nephrology and Hypertension, Department of Internal Medicine, Iwate Medical University School of Medicine, Yahaba, Japan

6 Okinawa Heart and Renal Association (OHRA), Naha, Okinawa, Japan

7 Department of Hemovascular Medicine and Artificial Organs, Faculty of Medicine, University of Miyazaki, Miyazaki, Japan

8 Division of Clinical Nephrology and Rheumatology, Niigata University Graduate School of Medical and Dental Science, Niigata, Niigata, Japan 
(a)

M0 : Markov 0 model (neither IgAN nor bladder cancer)

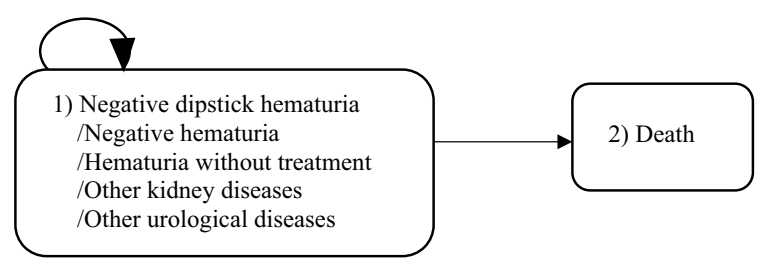

(b)

(M) : Markov IgAN model

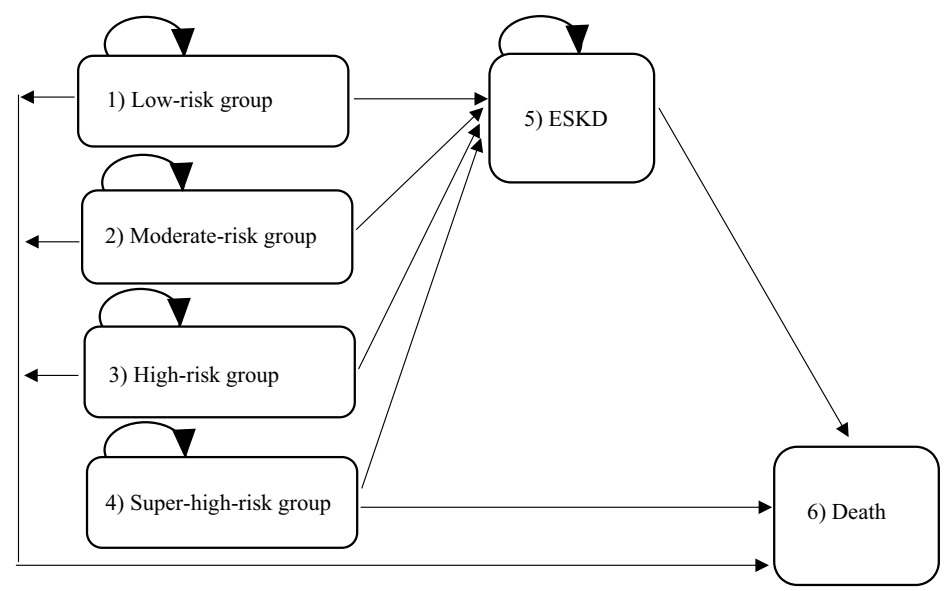

(c)

M $\mathrm{BC}$ : Markov bladder cancer model

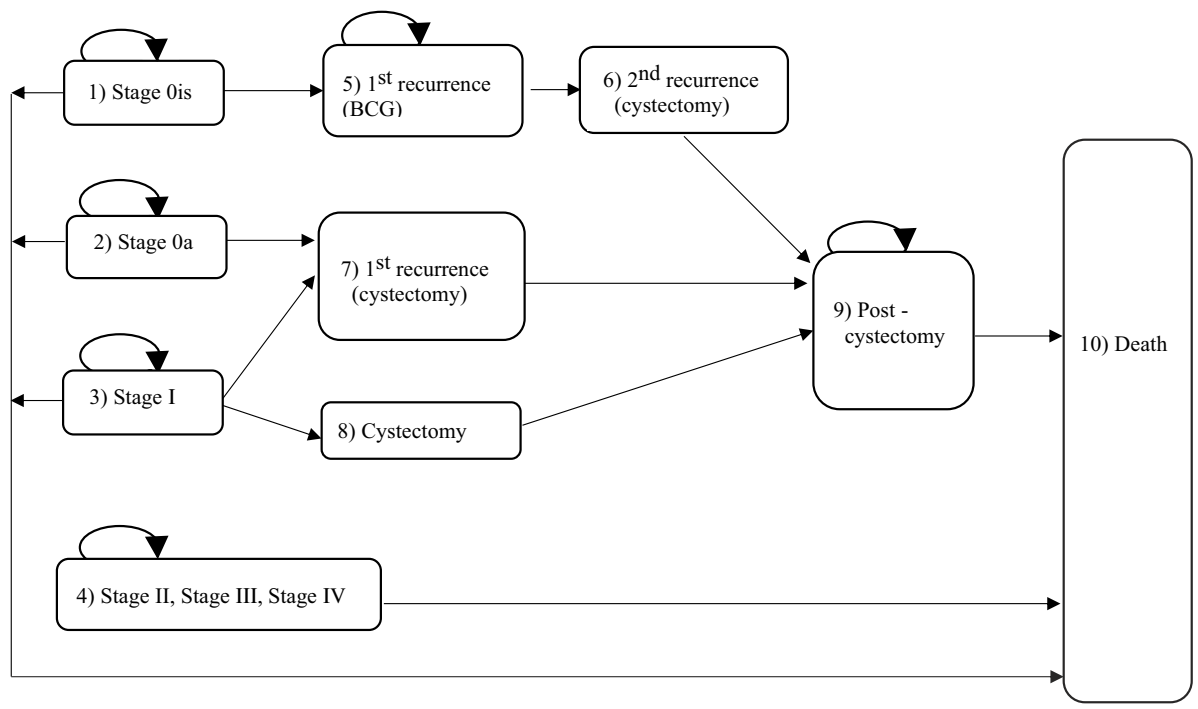

